\title{
Ideología, cientificismo y neutralidad: una reconsideración critica de la economía desde una perspectiva marxiana- gramsciana
}

\author{
Roberto Lampa ${ }^{1}$ y Nicolás Hernán Zeolla ${ }^{2}$
}

Resumen. La construcción de conocimiento dentro de la economía mainstream u ortodoxa considera a la abstracción lógica de los supuestos, la formalización de los razonamientos y la enunciación de hipótesis contrastables a partir de la efectividad de sus predicciones como garantía de neutralidad investigativa y rigurosidad científica. Sin embargo, como cualquier otra propuesta metodológica sobre la construcción de conocimiento científico dista de ser neutral. A partir del concepto de ideología en Karl Marx y Antonio Gramsci es posible comprender que la metodología adoptada en la ciencia económica es el resultado de un proceso histórico cuyo objetivo fue dar forma a una teoría capaz de establecer un nuevo sentido común económico que legitimara las necesidades del bloque histórico dominante. Su origen puede rastrearse desde el surgimiento de la revolución marginalista a fines del siglo XIX, pasando por la matematización de la disciplina en la segunda posguerra.

Palabras clave: Marx; Gramsci; Ideología; Método Científico.

Códigos JEL: B41; A11; b14

\section{[en] Ideology, scientism and neutrality: a critical reconsideration of economics from a Marxian-Gramscian perspective}

\begin{abstract}
The construction of knowledge within the mainstream or orthodox economy considers that the logical abstraction of assumptions, the formalization of reasoning and the enunciation of testable hypotheses are based on the effectiveness of their predictions as a guarantee of research neutrality and scientific rigor. However, like any other methodological proposal on the construction of scientific knowledge, it is far from neutral. From the concept of ideology in Karl Marx and Antonio Gramsci it is possible to understand that the methodology adopted in economic science is the result of a historical process whose objective was to give shape to a theory capable of establishing a new economic common sense that would legitimize the needs of the dominant historical block. Its origin can be traced from the emergence of the marginalist revolution in the late nineteenth century, through the mathematization of the discipline in the second postwar period.
\end{abstract}

Keywords: Marx; Gramsci; Ideology; Scientific methodology.

JEL Codes: B41; A11; b14

Sumario. 1. Introducción. 2. Marx y la crítica de la ideología económica. 3. Profundizando la crítica marxiana: ideología y teoría económica desde la perspectiva de Antonio Gramsci. 3.1. El concepto gramsciano de ideología. 3.2. La reflexión Gramsciana como crítica de la economía (marginalista). 4. ¿La economía contemporánea como ideología? 4.1. La metodología de la teoría económica mainstream. 4.2. La crítica marxiana: Marx y Gramsci. 5. Conclusiones. 6. Referencias.

Cómo citar: Lampa, R. y Hernán Zeolla, N. (2018) Ideología, cientificismo y neutralidad: una reconsideración critica de la economía desde una perspectiva marxiana-gramsciana, en Iberian Journal of the History of Economic Thought $\mathrm{n}^{\mathrm{o}} 5.2,133-145$.

Roberto Lampa. Investigador Designado CONICET (Consejo Nacional de Investigaciones Científicas y Técnicas); Profesor Asistente del Centro de Estudios Económicos del Desarrollo, Instituto de Altos Estudios Sociales, Universidad Nacional de San Martín, Buenos Aires, Argentina. Autor Correspondiente: roberto.lampa@conicet.gov.ar

2 Nicolás Hernán Zeolla. Becario doctoral CONICET (Consejo Nacional de Investigaciones Científicas y Técnicas); Investigador del Centro de Estudios Económicos del Desarrollo, Instituto de Altos Estudios Sociales, Universidad Nacional de San Martín, Buenos Aires, Argentina. 


\section{Introducción}

Para la economía convencional, el carácter científico de la disciplina viene dado por la adopción de un método que considera la abstracción lógica y la formalización como criterios objetivos de rigor científico en el proceso de construcción del conocimiento económico.

Sin embargo, tal nivel de abstracción conlleva el riesgo de una excesiva simplificación de la realidad, omitiendo la capacidad explicativa de los postulados básicos de la teoría. Por otro lado, el abordaje epistemológico adoptado solo exige la elaboración de teorías que muestren poder predictivo, es decir, sean verificables con la experiencia pasada o futura. Pero, así y todo, en muchos casos la falta de una contrastación empírica de la teoría no es motivo suficiente para desecharla, ya que en el fondo aún resulta lógicamente consistente. En este sentido, aparecen muchos interrogantes respecto a la metodología adoptada y el tipo de saber convencional que produce la disciplina económica.

A raíz de esta premisa, el objetivo del presente trabajo será analizar de una manera crítica la supuesta neutralidad del método adoptado por la teoría económica dominante. En especial, partiendo de los trabajos de Marx y Gramsci sobre la indisoluble relación entre ideología y análisis económico, el trabajo buscará cuestionar el límite que impone la metodología adoptada a la verificación empírica como criterio de selección teórica, el carácter acumulativo de la construcción de conocimiento y la matematización como herramienta y fin en sí misma.

La hipótesis del trabajo será que la metodología adoptada por la teoría económica $m a-$ instream no es un elemento neutral que busca comprender los fenómenos económicos tal cual son, sino que ella es el resultado un proceso histórico cuyo objetivo (ideológico) es la construcción de un sentido común económico que sirva como herramienta para legitimar la organización social de la producción vigente.

El trabajo estará dividido en cuatro secciones, además de esta introducción. En la sección 2 presentaremos el análisis de Marx de la relación entre ideología, economía política burguesa y economía vulgar. Luego, en la sección 3, introduciremos algunos elementos de la teoría gramsciana en torno al problema de la ideología, la ciencia y la teoría económica. A partir de allí, en la sec- ción 4 analizaremos críticamente los principales aspectos metodológicos de la teoría económica dominante (como el carácter predictivo, la objetividad, la acumulación del conocimiento, la formalización...) haciendo uso del concepto marxiano y gramsciano de ideología. Por último, en la sección 5 esbozaremos las conclusiones.

\section{Marx y la crítica de la ideología económica}

A partir de las Tesis sobre Feuerbach, los problemas de la ideología y del papel desempeñado por los intelectuales en las sociedades capitalistas asumen una importancia central en el proyecto científico marxiano.

Como destaca Labica (1987), la Tesis decimoprimera cumple un papel clave, en este sentido:

"Los filósofos no han hecho más que interpretar de diversos modos el mundo, pero de lo que se trata es de transformarlo" (Marx, 2015[1845])

Esta celebre cita se puede legítimamente interpretar como un programa de investigación en desarrollo por parte de Karl Marx en los sucesivos cuarenta años. Sin embargo, un hecho sobresaliente es que dicho programa se caracteriza por la explicita oposición entre el conocimiento genuino de la sociedad capitalista, por un lado, y la representación (falaz) que los filósofos han dado de dicho fenómeno, por el otro lado. En este sentido, Marx condiciona la misma posibilidad de comprensión y, por ende, de transformación de la realidad social y económica a un salto de paradigma por parte del investigador, el cual tiene necesariamente que abandonar las viejas creencias idealistas para adoptar una nueva postura que podríamos definir teórico-práctica, es decir, saber conjugar teoría y praxis (análisis abstracto e histórico).

A partir de esta premisa, un año más tarde Marx y Engels desarrollan el tema más en profundidad en la Ideología Alemana. En primer lugar, se introduce una relación bidireccional entre formas de la realidad y formas de la conciencia: dado que el hombre actúa como un traité d'union entre mundo exterior y mundo interior, todas las formas de conciencia son históricas y no es posible separar la esfera material de la esfera intelectual. Por ende, todo 
pensamiento humano es concebido por Marx y Engels como parte integrante de la realidad y no abstracción de la misma: "No es la conciencia lo que determina la vida, sino que es la vida lo que determina la conciencia" (Marx y Engels, 1959 [1846])

Sin embargo, en la sociedad capitalista la división entre trabajo manual e intelectual no permite tomar conciencia de semejante relación (Sprinzak, 1975). Por un lado, la producción material se emancipa completamente de los hombres, a pesar de ser el producto de la acción humana. Y, por otro lado, la producción intelectual se separa por completo de las actividades materiales. El intelectual se convierte entonces en ideólogo: en pos de permitir a los seres humanos de reconciliar formas de conciencia y formas de la realidad, reproducirá las contradicciones de la conciencia y les dará legitimidad. No solo los ideólogos invierten la relación entre formas materiales y abstractas (asumiendo que las grandes ideas determinan y plasman a la realidad) sino que justifican dicha inversión real en base de supuestas leyes naturales, que en cuanto tales, son también incontrovertibles. Llamativamente, a la hora de identificar una categoría de intelectuales que se destacan por su actitud ideológica Marx elije a los economistas. Al igual que los filósofos alemanes - que, por medio de las categorías del pensamiento puro, pretendieron explicar algo que todavía no existía en Alemania, es decir, el capitalismo y el estado burgués - los economistas reducen todas las múltiples relaciones entre los hombres a una sola relación -que Marx define "la teoría de la utilidad"convirtiendo su análisis en una mera apología del estado de cosas presente (Drucker, 1972). Enfatizando que dicha relación beneficiaría indistintamente a todos los miembros de la sociedad, Marx añade:

"La ciencia en que tiene su verdadero asiento esta teoría de la utilidad es la economía; (...) explica todas las actividades de los individuos en sus relaciones mutuas como una relación de utilidad y utilización, entre otras, por ejemplo, el lenguaje, el amor, etc. (...) aquellas relaciones no valen para los individuos en función de ellas mismas, como su propia manifestación, sino más bien como formas o disfraces, no de la categoría de la utilización, en modo alguno, sino de un tercer fin o de una tercera relación real, que recibe el nombre de relación de utili- dad. El carnaval en el lenguaje sólo tiene sentido cuando es expresión inconsciente o consciente de un carnaval real. Y, en este caso, la relación de utilidad encierra un sentido perfectamente determinado, a saber, el de que yo hago algo útil para mí al menoscabar a otro (exploitation de l'homme par l'homme)." [...] "El contenido económico va convirtiendo poco a poco la teoría de la utilidad en una simple apología del orden existente, en la demostración de que, en las condiciones existentes, las relaciones actuales entre los individuos son las más beneficiosas de todas y las que más benefician a la comunidad. Y éste es el carácter que dicha teoría presenta en todos los economistas modernos." (Marx y Engels, 1959 [1846], Cap. 2, Parte III, La Economía del Nuevo Testamento)

A partir de esta formulación pionera, podemos entonces llegar a una primera conclusión. Para Marx la ideología está indisolublemente ligada a la estructura de clases de la sociedad capitalista. A pesar del uso de un método equivocado para la investigación social, los ideólogos del capitalismo - es decir los economistas - logran mistificar y distorsionar la comprensión de la sociedad por parte de las masas (Nielsen, 1989). De esa forma, las ideas de las clases dominantes se vuelven ideología dominante en la sociedad gracias al papel crucial que cumplen los intelectuales y, en particular, los economistas.

Si bien las conclusiones de la Ideología Alemana mantienen su vigencia en su forma inicial, en los años siguientes la mirada de Marx se hace más articulada. Por ejemplo, en los Grundrisse de 1857-58 Marx introduce unas importantes diferencias entre economistas.

Allí, Marx reafirma su juicio crítico hacia la actitud ideológica de la mayoría de los economistas de su época, a la vez que reconoce a Adam Smith una clara superioridad metodológica, y por ende científica, sobre los demás economistas vulgares. Marx considera que el principal mérito de Smith está representado por su capacidad de conjugar análisis abstracto e histórico: por ejemplo a la hora de considerar el problema de la división del trabajo, Smith remite a un tipo histórico de trabajo (el trabajo asalariado, típico del capitalismo), si bien en el marco de un análisis abstracto. De esa manera, Smith asume implícitamente que todas las categorías analíticas, aunque abstractas, son el producto de relaciones sociales históricamen- 
te determinadas. En consecuencia, el alcance "científico" del trabajo de Smith es inconmensurablemente superior a lo de sus contemporáneos.

Sin embargo, aun así, los economistas burgueses como Smith caen en otro error para Marx, más sutil pero igual de ideológico: asumir que el capitalismo sea la forma perfecta y eterna de organizar las relaciones sociales de producción. De manera inversa, los resultados del análisis económico tienen plena vigencia solo en el marco de dichas relaciones capitalistas y para estas relaciones. Siguiendo a Marx:

“...incluso las categorías más abstractas, a pesar de su validez precisamente debida a su naturaleza abstracta para todas las épocas, son no obstante, en lo que hay de determinado en esta abstracción, el producto de condiciones históricas y poseen plena validez sólo para estas condiciones y dentro de sus límites." (Marx, 1989 [1857], p. 55)

Dicho sencillamente, ninguna teoría de la sociedad puede ser cientificamente verdadera, es decir, universalmente valida, independientemente del contexto histórico, geográfico, social y político. El verdadero objetivo del economista debe ser proveer un análisis históricamente relevante. $Y$ es en este sentido que las obras de Smith tenían un alto valor científico en su época. Podríamos decir que en Marx esto se debe básicamente a tres motivos. En primer lugar, porque investigaban relaciones sociales y no ideas abstractas como Hegel y los filósofos alemanes. En segundo lugar, porque no quedaban circunscritos a la manifestación superficial de los fenómenos sociales, como los economistas vulgares. En tercer lugar, porque no tenían un perfil abiertamente ideológico y partidario, sino que se proponían, como punto de departida, el alcance de objetivos neutrales y "científicos" (Parekh, 1982). Es por ello que, a juicio de Marx, los trabajos de los economistas vulgares, como Bastiat o Malthus, quedaron siempre relegados en un plan exclusivamente ideológico ${ }^{3}$.

\footnotetext{
De todas maneras, cabe aclarar que también el valor "científico" de la obra de Smith no es absoluto para Marx, sino limitado al estudio de una particular etapa del capitalismo. En este sentido, reivindicar sic et simpliciter los trabajos de Smith hoy en día seria no solo un ejercicio retorico, sino meramente ideológico y vulgar en la lógica marxiana (Drucker, 1972).
}

Sin embargo, debemos considerar que para Marx no puede existir una "ciencia económica pura". Y es en este sentido que los trabajos de Smith contenían un prejuicio ideológico burgués, al suponer que el capitalismo era la forma final de modo de la producción, y terminan reproduciendo en el ámbito económico la manera de pensar de aquellos creyentes que ven todo lo que queda afuera de su religión como necesariamente falso (Marx, 1894).

De modo que para Marx todo tipo de investigación científica tiene tanto una premisa ideológica como una interpretación filosófica. Por lo tanto, ciencia e ideología son inseparables. Sin embargo, esto no implica que el análisis económico y social no pueda contener una parte abstracta, es decir, independiente de la arbitrariedad de las premisas. Es por esto que Marx definía las teorías de Smith y Ricardo como "economía política burguesa" para destacar que en ellas podíamos encontrar elementos imparciales pero adentro de un marco parcial; una mirada desinteresada si y un método riguroso, pero no tanto como alcanzar resultados plenamente científicos (Parekh, 1982).

En este sentido, Marx no concibe la relación entre ciencia e ideología en términos dicotómicos, si bien enfatiza la necesidad de un núcleo del análisis económico independiente de la arbitrariedad de las premisas, la crítica marxiana no apunta a definir ningún "criterio abstracto de cientificidad". Ya que Marx reconoce que todo tipo de investigación está necesariamente envuelta en ideología, y es por esto que es posible rechazar a priori todo tipo de cientificismo (Larrain, 1982).

En este sentido [repetición], para Marx la obsesión cientificista es una prerrogativa de los economistas vulgares, dada su reiterada insistencia sobre la naturalidad o racionalidad tanto de las leyes económicas como del orden social y económico presente.

En línea con lo anterior, en las Teorías sobre la Plusvalía y en El Capital Marx delinea una relación inversa entre "contenido científico" de la economía e intensidad del conflicto de clases. Para este, tanto la "cientificidad" de la economía política burgués como la redundancia ideológica de la economía vulgar representan dos fenómenos ligados indisolublemente. En línea con esto es que Marx considera que Smith y Ricardo elaboraron teorías científicamente superiores ya que sus trabajos vieron la luz en un contexto políticamente favorable, caracterizado por el dinamismo capitalista contra el viejo régimen. De modo 
que una fuerte caracterización ideológica hubiese sido innecesaria dadas la relaciones sociales de producción "normalizadas". Sin embargo, a medida que el conflicto de clases se profundiza y sacude a las sociedades europeas, la teoría económica se aleja paulatinamente de la realidad como defensa del estatus quo (Grossman, 1977; Maguire, 1976):

"A medida que la economía tiende a hondar, no solo se plantea contradicciones, sino que se alza frente a ella su propia contradicción, al paso con el desarrollo de las contradicciones reales [que se contienen] en la vida económica de la sociedad. A medida que esto ocurre, la economía vulgar se toma conscientemente apologética y trata de eliminar forzadamente, con sus charlatanerías, los conceptos y las contradicciones correspondientes" (Marx, 1863, p.444)

Desde esa perspectiva, el mismo proyecto científico de Marx puede ser legítimamente interpretado como una "ciencia del proletariado", es decir una respuesta a la definitiva e irreversible vulgarización de la economía política sucesivas a los acaecimientos de 1848 y de la separación, más profunda, entre política y economía (Balibar, 1988).

El siguiente párrafo del Tomo III de $\mathrm{El} \mathrm{Ca}$ pital resume nuestro análisis con extrema claridad:

“...el mundo encantado, invertido y puesto de cabeza donde Monsieur le Capital y Madame la Terre rondan espectral mente como caracteres sociales y, al propio tiempo de manera directa, como meras cosas. El gran mérito de la economía clásica consiste en haber disuelto esa falsa apariencia, esa superchería, esa autonomización recíproca y ese esclerosamiento de los diferentes elementos sociales de la riqueza, esa personificación de las cosas y cosificación de las relaciones de producción, esa religión de la vida cotidiana (...) No obstante, incluso sus mejores portavoces, como no podía ser de otra manera desde el punto de vista burgués, siguen siendo prisioneros, en mayor o menor medida del mundo de la apariencia críticamente disuelto por ellos, y por ende todos incurren más o menos en inconsecuencias, semiverdades y contradicciones no resueltas. (...) Por eso es asimismo natural que la economía vulgar, que es nada más que una traducción didáctica, más o menos doctrinaria, de las representaciones corrientes de los agentes reales de la producción, entre las cuales introduce cierto orden inteligible, encuentre precisamente en esa trinidad, donde está extinguida toda la conexión interna, la base natural, y puesta al abrigo de toda duda, de sus triviales jactancias. Esa fórmula corresponde al mismo tiempo al interés de las clases dominantes, puesto que proclama la necesidad natural y la legitimación eterna de las fuentes de sus entradas, elevándolas a la calidad de dogma. Al exponer la cosificación de las relaciones de producción y su autonomización frente a los agentes de la producción, no entramos a analizar la manera en que las conexiones a través del mercado mundial, sus coyunturas, el movimiento de los precios de mercado, los períodos del crédito, los ciclos de la industria y el comercio, la alternancia de la prosperidad y la crisis, se les presentan como leyes naturales todopoderosas que los dominan al margen de su voluntad y se imponen frente a ellos como una ciega necesidad." (Marx y Engels, 2009 [1894], Vol. III, cap. 48)

En este sentido, podemos afirmar que a lo largo de su programa de investigación, Marx desarrolla con creciente claridad una crítica al papel que la ideología cumple en los trabajos de los economistas de la época. Rechazando todo cientificismo, Marx reconoce que es inevitable que la ideología influencie tanto las premisas como las interpretaciones del análisis económico, pero define un núcleo central del análisis social y económico libre de condicionamientos ideológicos en cuanto abstracto y a la vez históricamente determinado, es decir, basado en la evidencia empírica. A diferencia de los economistas vulgares, Marx destaca que cada intento de asimilar la economía a una ciencia natural es en realidad la demonstración máxima del papel negativo que la ideología puede ejercer sobre esta disciplina. Desde este punto de vista, a partir de la categoría marxiana de economistas vulgares es posible observar que son estos los que más se han caracterizado por el uso/abuso de leyes naturales a la hora de describir el funcionamiento de las economías capitalistas.

\section{Profundizando la crítica marxiana: ideología y teoría económica desde la perspectiva de Antonio Gramsci}

\subsection{El concepto gramsciano de ideología}

Al igual que Marx, el proyecto filosófico de Antonio Gramsci también tiene su punto de parti- 
da en la tesis decimoprimera sobre Feuerbach o, mejor dicho, de la crítica a la interpretación de dicha Tesis popularizada por el filósofo italiano Benedetto Croce (Labica, 1987). A la visión crociana - que interpretaba la onceava Tesis como un remplazamiento de la filosofía por la acción práctica - Gramsci contrapone una lectura más afin a la de Marx enfatizando la necesidad de una redefinición del papel del investigador social como aquel capaz de conjugar teoría y praxis.

En oposición explicita a la filosofia de la historia de Croce, Gramsci define su proyecto como filosofia de la praxis: un análisis filosófico destinado a la comprensión y transformación de la sociedad capitalista, en particular de Europa Occidental. Desde esta perspectiva, la filosofía de la praxis que propone Gramsci es un enfoque metodológico al mismo tiempo que una herramienta de construcción dentro de la lucha política.

Para Gramsci, no existe la "filosofía" en general, sino que existen diversas filosofías o concepciones del mundo y en el "obrar" o la praxis se desata una disputa por cuál va a prevalecer. Sin embargo, la disputa no tiene un carácter permanente, sino que una vez establecida una forma de pensamiento, esta se transforma en una premisa implícita, subyacente al proceso de producción y reproducción social. En este sentido, para Gramsci el carácter más alto de concepción del mundo es la ideología y su influencia está implícita en todos los ámbitos de la vida en general, desde " $e l$ arte, el derecho, la actividad económica y en todas las manifestaciones de la vida individual y colectiva" (Gramsci, 1971:12).

Es en este sentido que Gramsci rechaza aquellas interpretaciones del materialismo histórico marxiano - típicas de la II Internacional - que se caracterizaban por su ultra-determinismo y sus contaminaciones Positivistas (Lampa, 2010). A partir de su célebre escrito de 1917 "La Revolución contra El Capital' Gramsci critica aquellos economistas italianos que habían provisto una lectura de Marx en la cual la ideología se reducía a mero reflejo de las transformaciones de la estructura subyacente o base material. En cambio, el análisis de la sociedad capitalista es algo más complejo: si bien las tendencias se encuentran vigentes en la estructura, sus consecuencias no tienen por qué realizarse necesariamente y mecánicamente en un plan político ${ }^{4}$.

\footnotetext{
La publicación de los Grundrisse han mostrado, si bien de manera póstuma, la validez de la interpretación Gramsciana previa: "En la producción social de su vida los hombres establecen determinadas relaciones necesarias e independientes de su voluntad, relaciones de producción
}

Sin embargo, Gramsci no se limita a releer Marx, sino que profundiza y radicaliza la postura marxiana, en particular a la hora de definir el papel de la filosofía.

Es en este contexto donde aparece la categoría de "bloque histórico" como un conjunto complejo, contradictorio y discorde de relaciones necesarias y reciprocas entre estructura, $o$ relaciones sociales de producción, y superestructura, o sistema de manifestación

La reciprocidad entre estructura y superestructura es clave para comprender la estrecha relación entre conocimiento, conciencia, ideología y praxis. Para Gramsci el conocimiento es una forma de influir en la conciencia teórica. Sin embargo, esta conciencia teórica no es estrictamente igual a la conciencia práctica, más ligada a aspectos permanentes de las relaciones estructurales.

En este sentido, la ideología es el punto más alto de esta conciencia teórica. Es decir, cuando esta conciencia se internaliza, se hace implícita, impone una unidad de acción del mundo coherente y unitaria. De esa manera, la ideología condiciona a un grupo social determinado, influye sobre su conducta moral, la dirección de su voluntad, eliminando toda capacidad de transformación del orden vigente. Sin embargo, esta relación no es permanente. Existen espacios de influencia que se presentan como disputas de hegemonías políticas, primero en el campo de ética individual, luego el de la política o el accionar colectivo. Por ello, para Gramsci las ideologías son concepciones del mundo en disputa. El conflicto llega a su punto más alto cuando esta nueva conciencia teórica busca la unidad con la práctica, desatando una crisis orgánica que busca modificar el orden establecido.

$\mathrm{Y}$ es en la difusión de estas nuevas concepciones del mundo tienen un rol clave los intelectuales. En la definición de Gramsci los intelectuales son:

"los «empleados» del grupo dominante para el ejercicio de las funciones subalternas de la he-

\footnotetext{
que corresponden a una fase determinada de desarrollo de sus fuerzas productivas materiales. El conjunto de estas relaciones de producción forma la estructura económica de la sociedad, la base real sobre la que se levanta la superestructura jurídica y política y a la que corresponden determinadas formas de conciencia social. El modo de producción de la vida material condiciona el proceso de la vida social política y espiritual en general. No es la conciencia del hombre la que determina su ser sino, por el contrario, el ser social es lo que determina su conciencia". (Marx, 1857)
} 
gemonía social y del gobierno político, a saber: 1) del consenso espontáneo que las grandes masas de la población dan a la dirección impuesta a la vida social por el grupo social dominante, consenso que históricamente nace del prestigio (y por lo tanto de la confianza) detentada por el grupo dominante, de su posición y de su función en el mundo de la producción"(Gramsci, 1960).

De este modo, la sociedad civil es el ámbito en el cual las clases dominantes logran consolidar su poder y donde difunden su visión del mundo. Entonces, la ideología es un momento más elevado de transmisión de una filosofía; es a la vez una moral y un conjunto de costumbres que tienen como fin el reconocimiento de la capacidad de una clase social de ejercer su dominación sobre el resto, ya que estos postulados se tornan implícitos y terminan constituyendo un nuevo sentido común.

Sin embargo, no todo es representación en la producción de hegemonía, consenso o dirección no coactiva sino que existe un sustento objetivo en la que se basa la capacidad del grupo dirigente de ejercer el control directo sobre el núcleo rector de la actividad económica, que desarrolla un papel clave (Thwaites Rey, 2007).

Respecto a la relación entre conocimiento científico e ideología, para Gramsci la ciencia tiene un doble carácter. Por un lado, la ciencia es la "base de la vida" la cual puede "limpiar los ojos de toda ilusión ideológica y colocar al hombre frente a la realidad, tal como ésta es" (Gramsci, 1971:63). Sin embargo:

"la ciencia es también es una superestructura, una ideología (...) la ciencia no se presenta jamás como desnuda noción objetiva; aparece siempre revestida de una ideología y, concretamente, la ciencia es la unión del hecho objetivo con una hipótesis o un sistema de hipótesis que superan el mero hecho objetivo" (Gramsci, 1971: 63-64).

De modo que la concepción Gramsciana de la ciencia radicaliza la postura marxiana. $\mathrm{Si}$ bien Marx negaba la existencia de una "ciencia pura", al mismo tiempo reconocía la posibilidad de hacer unos análisis cualitativamente superiores, dados ciertos requisitos. En cambio, Gramsci parece extender al ámbito inmaterial la crítica al Capital, sugiriendo que también en este ámbito la inversión real sujeto/objeto es una llave de lectura consistente. Tal como en la estructura económica se invierte la relación entre sujeto (hombre productor) y objeto (mercancía) en virtud del fetichismo de la mercancía, asimismo en la superestructura la conformidad al método científico (que es un reflejo de la ideología y, por ende, objeto de la actividad humana) y a las leyes naturales se impone como regla inderogable para ordenar la vida de los hombres (sujetos). La cientificidad, por ende, se convierte en un fetiche en este plan del análisis.

\subsection{La reflexión Gramsciana como crítica de la economía (marginalista)}

A lo largo de muchos años los historiadores del pensamiento económico - con la excepción de Lunghini (1994) - se han limitado a enfatizar los errores de Gramsci en el ámbito económico y su substancial inutilidad para nuestra disciplina (Faucci, 2010) o a reconstruir su influencia, en un plan meramente filosófico, sobre Piero Sraffa y Ludwig Wittgenstein (Davis, 2002; Sen, 2003).

Sin embargo, en épocas más recientes esta tendencia se ha revertido: también gracias a la publicación de los manuscritos inéditos de Piero Sraffa, el estudio de la crítica gramsciana a la economía se ha profundizado (Leopold, 2013; De Vivo, 2017).

En particular, al igual que la crítica sobre aspectos filosóficos, la cuestión de la ideología en Gramsci también nos da algunos elementos para cuestionar las transformaciones teóricas de la ciencia económica.

En términos generales, para Gramsci el capitalismo es el primer modo de producción que se caracteriza por la neta separación entre esfera política y económica. A partir de la célebre representación del Tableau Économi$q u e$, la actividad económica se vuelve un fin a sí misma. Esta separación le da el origen a la economía como disciplina científica, dada la necesidad de tener una ciencia del capitalismo. Por lo tanto, desde el principio la economía se caracteriza por su intento de imitar las ciencias naturales, adoptando un método abstracto (el método del "supuesto que", en las palabras de Gramsci) y la lógica deductiva.

Para Gramsci, David Ricardo es el verdadero padre de la economía y, por intermedio de la crítica de la economía política de Marx, 
guarda cierta relación su filosofía de la praxis. Llamativamente, a juicio de Gramsci la influencia ricardiana no se debe tanto a los desarrollos de la teoría del valor sino a su enfoque abstracto, para Gramsci:

\section{"el método de 'dado que' de la premisa, a la cual se sigue cierta consecuencia, parece que debe ser identificado como uno de los puntos de partida (de los estímulos intelec- tuales) de las experiencias filosóficas de la filosofía de la praxis" (Gramsci, 1971:108)}

Sin embargo, la abstracción ricardiana es aceptable por Gramsci porque es también "históricamente determinada". De modo coherente con el punto de vista marxiano, también Gramsci está convencido que el único método de investigación correcto es aquel que logra conjugar historicismo, anti determinismo y abstracción, es decir, tener como objeto de estudio un "mercado determinado" que no equivalga a una abstracción pura:

\begin{abstract}
"Occorre fissare il concetto di mercato determinato. Come viene assunto nell' economia «pura» e come nell'economia critica. Mercato determinato nell'economia pura è una astrazione arbitraria, che ha un valore puramente convenzionale ai fini di un'analisi pedantesca e scolastica. Mercato determinato per l'economia critica sarà invece l'insieme delle attività economiche concrete di una forma sociale determinata, assunte nelle loro leggi di uniformità, cioè «astratte», ma senza che l'astrazione cessi di essere storicamente determinata"s(Gramsci, 1929-1930)
\end{abstract}

En cambio, el nuevo paradigma marginalista, detrás de una fachada de seriedad y cientificidad esconde una dimensión ideológica prevalente, a raíz de la cual la denominación de la ciencia moderna como economics se parece a la teología:

\footnotetext{
"Es necesario aclarar el concepto de mercado determinado. Así como es concebido por la "economía pura" y la "economía critica". En la economía pura mercado determinado es una abstracción arbitraria que tiene un valor meramente convencional, finalizado a un análisis escolástico. Para le economía critica mercado determinado será en cambio el conjunto de las actividades económicas concretas de una forma social determinada, concebidas en el marco de su leyes de conformidad, es decir abstractas pero sin que la abstracción cese de ser históricamente determinada." (Traducción propia)
}

"Si può domandare se l'economia pura sia una scienza oppure se essa sia «un qualche cosa d'altro» che però si muove con un metodo che in quanto metodo ha un suo rigore scientifico. Che esistano attività di questo genere è mostrato dalla teologia. Anche la teologia parte da una certa serie di ipotesi e quindi costruisce su di esse tutto un massiccio edifizio dottrinale saldamente coerente e rigorosamente dedotto. Ma la teologia è perciò una scienza?"'(Ibidem)

Por ende, Gramsci no comparte la idea de Croce de que el conocimiento avanza siempre con un carácter acumulativo ${ }^{7}$ y que, por lo tanto, el nuevo paradigma marginalista se merece el título de "economía pura" por su superioridad científica sobre la filosofía de la praxis.

En principio, uno podría pensar que la idea del progreso del conocimiento podría ser evidente en las ciencias naturales, si entendemos el avance como una "complejización instrumental", el desarrollo de nuevas técnicas para mayor percepción del mundo, etc. (Gramsci, 1971:143-144). Sin embargo, dice Gramsci, incluso en las ciencias naturales el sentido de este avance no puede estar disociado de las relaciones de producción subyacentes ya que toda ciencia está ligada a las necesidades de la vida y la actividad del hombre, de allí el carácter ideológico e histórico del conocimiento científico.

En la misma línea, refiriendo a la teoría económica, debido a la recurrencia de crisis y problemas sociales la noción de progreso en el conocimiento no parecería tan evidente. En economía las relaciones de producción y su dinámica son un insumo en la investigación. Por ello, y de manera fundamental, el sentido que se le da a estos hechos históricos forma parte constitutiva de la disputa por la hegemonía y la construcción de legitimidad de la clase dominante. Siguiendo a Gramsci, a partir de transformaciones en la organización social de la producción "se multiplican los programas

\footnotetext{
"Nos podríamos preguntar si la economía pura es una ciencia o es otra cosa, pero caracterizada por un método que en cuanto método tiene cierto rigor científico. La existencia de semejantes actividades por ejemplo es demostrada por la teología. La teología también supone un conjunto de hipótesis y luego construye a partir de ellas un imponente edificio teórico fuertemente coherente $y$ deducido rigurosamente. Pero acaso consideramos que la teología es una ciencia por eso?" (Traducción propia)

Este punto de vista se ha impuesto definitivamente en el segundo posguerra, reflejo de la postura verificacionista (hipotético-deductiva) (Friedman, 1953; Machlup, 1978)
} 
teóricos que exigen ser justificados de manera realista en cuanto demuestran ser asimilables por los movimientos prácticos, que solo así se tornan más prácticos y reales" (Gramsci, 1971:46). En este sentido, la teoría económica aparece como una representación que guarda estrecha relación con las fuerzas desencadenadas por los cambios en la base material, que a partir de cierto momento necesitan ser justificadas, de modo que las fuerzas rectoras puedan ser eficientes y expansivas.

Al mismo tiempo, para poder hablar de progreso en el conocimiento en una disciplina deberíamos poder circunscribir aquello que decimos conocer.

De manera parecida a Veblen (1898), para Gramsci la economía no es el análisis teórico de un entramado cerrado, sino que es la teoría sobre un proceso cuyas relaciones de causa efecto no son definitivas, por lo que el sentido de verdad no puede definirse de una manera mecánica.

A partir de aquí aparece la segunda cuestión crucial del análisis gramsciano sobre la economía: el análisis del exitoso caso estadounidense como producto de una poderosa ideología productivista.

Como punto de partida, Gramsci considera las transformaciones que sufrió la economía mundial debido a la crisis de los años Treinta. En Europa la respuesta fue la reacción, el Fascismo, en cambio en Estados Unidos fue la racionalización de la producción, con la profundización del taylorismo y del fordismo. Gramsci analiza esta cuestión en Fordismo y Americanismo e intenta comprender estas diferencias a partir de la configuración de la superestructura en uno y otro continente. De manera distintiva, Estados Unidos no tiene grandes tradiciones históricas y culturales, han heredado la moral puritana que tiene como característica la visión de que la vida pública debe ser consecuente con los actos privados y existe naturalmente una composición demográfica racional, por la ausencia de sectores sociales improductivos y rentistas. En Europa, en cambio, sucede lo contrario ya que la historia pasada ha dejado una cantidad de sedimentaciones pasivas: personal estatal, clero, comercio de rapiña, etc. que viven de actividades subalternas de la producción. Como resultado, en Estados Unidos, dice Gramsci: "ha sido relativamente fácil racionalizar la producción y el trabajo, combinando hábilmente fuerza (destrucción del sindicalismo obrero de base territorial) con la persuasión (altos salarios, beneficios sociales diversos, propaganda ideológica y política habilísima) y consiguiendo basar toda la vida del país sobre la producción. La hegemonía nace de la fábrica y no tiene necesidad de ejercerse más que por una cantidad mínima de intermediarios profesionales de la política y la ideología" (Gramsci, 1978:66).

En este sentido, para Gramsci, la característica relevante de la adaptación diferencial a la crisis es la capacidad de adaptación de la economía americana a la nueva organización de la producción. En Estados Unidos, la clase dominante solo tiene que construir hegemonía en la fábrica y la nueva racionalidad logra imponerse sobre toda la sociedad, sin necesidad de un gran desarrollo intelectual para que sea legitimada. De algún modo, podemos decir que en este caso es la estructura que se refleja inmediatamente y sin mediaciones sobre la superestructura, produciendo una ideología y una "american way of living" basadas sobre la acumulación y la espasmódica búsqueda de la ganancia personal.

\section{4. ¿La economía contemporánea como ideología?}

\subsection{La metodología de la teoría económica mainstream}

El método de construcción de conocimiento de la economía mainstream moderna se basa en la célebre propuesta metodológica de Friedman (Friedman, 1953). Por ende, sus bases epistemológicas se corresponden con el verificacionismo hipotético-deductivo (Hempel y Oppenheim, 1948). En este sentido, el criterio de demarcación de la ciencia económica implica la construcción de teorías que contengan hipótesis capaces de ser testeadas empíricamente. Sin embargo, la verificabilidad de la teoría no tiene que ver con la capacidad descriptiva de los postulados básicos sino con su capacidad predictiva.

En particular, a partir del trabajo de Friedman, el abordaje metodológico en economía considerará aspectos del verificacionismo como criterio de cientificidad de los postula- 
dos teóricos y un mayor grado de abstracción como elemento deseable de los postulados básicos.

Por otro lado, la relevancia descriptiva o el realismo de un postulado básico por sí mismo cesan de ser unos criterios suficientes para rechazar o aceptar una hipótesis entre otras. Para Friedman, una teoría es más simple y fructífera en la medida que necesita menor cantidad de hipótesis (realistas o no) para realizar predicciones más certeras.

Sin embargo, al igual que en otras disciplinas, el verificacionismo en economía tiene los mismos problemas que marca la tesis de Duhem-Quine. Si llevando adelante la verificación empírica se obtienen resultados contrarios a los esperados, la incompatibilidad entre teoría y realidad podrá siempre explicarse por la existencia de un postulado contrastable empíricamente (algún shock no considerado, un control ausente o un dato idiosincrático que no se ajusta al modelo general), lo que en definitiva mantiene el "core teórico" preservado. La forma de explicar la nueva evidencia es entonces incluir supuestos auxiliares o ad hoc que den cuenta de estos casos particulares sin contradecir la teoría abstracta más general.

La economía mainstream empieza así a transitar un terreno pantanoso: de no ser lo suficientemente evidente, cualquier resultado desfavorable que la teoría prevé y no se cumple, siempre puede atribuirse a un error artificis (error de su aplicación, es decir del economista en particular) y no a un error artis (error del arte, es decir de la teoría).

Al mismo tiempo, el mayor nivel de abstracción de los postulados básicos se vuelve de por sí un signo de mayor cientificidad y capacidad de generalización. En este sentido, se inscribe la matematización de la disciplina a partir de los años cincuenta, y la adopción de la representación de la dinámica económica con el herramental de la física termodinámica (Mirowski, 1989).

Siguiendo a Leijonhufvud (1973), en el mainstream económico moderno la matemática comienza entonces a ser tratada como un tótem, es decir un fin en sí misma que funciona como criterio de status entre los distintos economistas.

\subsection{La crítica marxiana: Marx y Gramsci}

Derivado del análisis de las secciones anteriores, Marx y Gramsci indican algunas líneas a partir de las cuales podemos encontrar res- puestas sobre los aspectos metodológicos pendientes en la ciencia económica convencional contemporánea.

En primer lugar, tanto Marx como Gramsci cuestionan el traspasamiento del método científico de las ciencias naturales en las ciencias sociales. Como planteo del problema científico en abstracto, la investigación debería orientarse a la búsqueda de leyes, líneas constantes, regulares y uniformes, vinculadas a una exigencia de resolver problemas prácticos de previsibilidad en el sentido explicito que tiene la definición de ciencia en las investigaciones físicas.

Sin embargo, este método aplicado a la "previsión de cuestiones históricas", como se utilizan en economía, no tiene sentido. Para Gramsci en particular, "realmente se 'prevé" en la medida en que se obra, en que se aplica un esfuerzo voluntario $y$, por lo tanto, se contribuye concretamente a crear el resultado 'previsto"' (Gramsci, 1971:143). La referencia directa de esto es el criterio de cientificidad adoptado por el mainstream económico y propuesto por Friedman (1953) y Machlup (1978). La previsión de los hechos históricos depende del obrar presente, esta históricamente condicionada y es incognoscible por definición. Por lo tanto, basar una metodología científica en estos criterios es un grave error conceptual. Como alternativa, tanto Marx como Gramsci sugieren una metodología que no niegue la dimensión ideológica de las ciencias sociales, sino que la circunscriba a las premisas y a la interpretaciones, por medio de un método abstracto pero históricamente determinado, es decir corroborado por la evidencia empírica.

En relación a ello, Gramsci también se detiene en problematizar la pretensión de fundar una metodología científica en la lógica puramente formal. La idea básica de esta crítica es que no existe el pensamiento "en abstracto", sino que cualquier representación esta históricamente condicionada. Este debate puede verse reflejado en el célebre intercambio entre Wittgenstein y Sraffa, donde se intuye con alguna claridad la influencia del pensamiento de Gramsci (Sen, 2003). La filosofía de Wittgenstein sostenía que la proposición y lo que ella describe deben tener la misma forma lógica, existiendo un estricto paralelismo entre lo real y el lenguaje. Sin embargo, y tras "frotarse los dedos debajo del mentón" (el gesto napolitano de "no me interesa") Sraffa le cuestiona la forma lógica que Wittgenstein le daría a ese 
gesto. El punto que se intenta presentar Sraffa allí es la manera en que las convenciones sociales pueden influir en el significado mediante expresiones y gestos, sin tener un correlato estricto entre una y otra.

En línea con lo anterior, la recurrencia a categorías abstractas o la tendencia a la matematización fue un aspecto creciente de la disciplina luego de la segunda guerra mundial. La intención del nuevo mainstream neoclásico (a partir de Samuelson) era asemejar a la dinámica económica a los modelos de termodinámicos de la física (Mirowski, 1989).

En primer lugar, la formalización tiene implícito la consideración de relaciones permanentes en los mercados que podríamos resumir como "invariancia de las relaciones estructurales". Esto podrá ser válido en ciencias naturales como la física o la biología. Sin embargo, en economía esto no es así. Siguiendo a Marx y Gramsci, desde la aparición de la crítica de la economía política sabemos que los mercados y su automatismo no son eternos, sino que son el resultado de una construcción histórica, lo que determina su caducidad al igual que la ciencia que la analiza (Gramsci, 1971:107; Marx, 1857)

A su vez, en términos del desarrollo de la matemática de los sistemas dinámicos, la matemática adoptada en economía es una matemática vetusta que incluso al momento de incorporarla en economía ya había resultado obsoleta (Mirowski, 1989). De esa manera, los modelos se han vuelto el moderno tótem de la tribu de los Econ (Leijonhufvud, 1973), es decir un fetiche que no siempre aporta un significativo avance en el conocimiento de la economía. Es difícil creer entonces que el objetivo fuera el avance científico de nuestra disciplina y no, como sugerirían Marx y Gramsci, una enorme operación ideológica finalizada a la marginalización de las teorías "heréticas" y a la defensa del estatus quo, en particular después de la enorme intensidad del conflicto de clases en el bienio 1968-69.

Igualmente, aunque este implícito, tampoco está de más decir que este giro hacia la formalización no fue una transformación natural. La construcción de un modelo implica la elaboración de una "realidad ficcional", que voluntariamente toma ciertos aspectos del fenómeno económico, pero intencionalmente desecha otros. En este sentido, con la matematización lo que se pierde es el realismo de los postulados, alejando a la disciplina de los aspectos históricos e institucionales.
Otro aspecto metodológico que podríamos cuestionar a partir de Marx y Gramsci es la objetividad del conocimiento científico. En términos generales, Gramsci considera la cuestión de si la ciencia puede dar la certeza de "la existencia objetiva de la realidad externa". En principio la realidad objetiva externa es un supuesto filosófico, sobre el que la ciencia no pueda dar pruebas. Sin embargo, la ciencia si puede ordenar el caos del mundo, seleccionado aquellos aspectos permanentes. En este sentido, el conocer implica elaborar conceptos nuevos para afinar los instrumentos de la experiencia. Esto es claro en las ciencias naturales, donde el desarrollo científico podría relacionarse estrictamente con el desarrollo mismo del instrumental de la experiencia y su verificación. Desde esta perspectiva la objetividad queda entendida como aquella experiencia que puede ser "verificada por todos los hombres, que es independiente de todo punto de vista ya sea meramente particular o de grupo" (Gramsci, 1971:62). Sin embargo, todo lo que la ciencia afirma no es objetivamente verdad de manera definitiva, ya que no es posible afirmar la existencia de la realidad como tal más allá de la relación con el hombre. Y como el hombre es devenir histórico, también lo es el conocimiento y la realidad y, por ende, para Gramsci la ciencia es una categoría histórica y una categoría en continuo desarrollo (Gramsci, 1971:151).

Una reflexión sobre estos aspectos es imprescindible y no implica necesariamente la aceptación de las teorías marxianas y gramscianas. En el mismo sentido, aunque desde otra perspectiva, Max Weber también cuestiona la objetividad del conocimiento científico. En la opinión de Weber la realidad no es siempre comprensible de manera directa sino que esta frecuentemente mediada por la subjetividad. Por ello, Weber solía distinguir entre juicios de valor y juicios de hecho, aclarando que "cualquier intento de analizar la realidad concreta es insignificante, ya sea porque la dirección de sus creencias personales, el reflejo de los valores en el prisma de su mente, es lo que direcciona su trabajo" (Weber, 1949).

\section{Conclusiones}

En este artículo propusimos, en primer lugar, una reconstrucción del pensamiento de Karl Marx sobre la relación entre ideología y teoría económica, en sus principales trabajos 
(Tesis sobre Feuerbach, Ideología Alemana, Grundrisse, Teorías sobre la Plusvalía, El Capital). Mostramos que en interpretación de Marx la ideología está indisolublemente ligada a la estructura de clases de la sociedad capitalista y los ideólogos del capitalismo es decir los economistas - logran mistificar y distorsionar la comprensión de la sociedad por parte de las masas. Además, aclaramos como, rechazando todo cientificismo, Marx reconoce que es inevitable que la ideología influencie tanto las premisas como las interpretaciones del análisis económico, pero define un núcleo central del análisis social y económico libre de condicionamientos ideológicos en cuanto abstracto y a la vez históricamente determinado, es decir corroborado por la evidencia empírica. A este método "virtuoso", Marx contrapone la asimilación de la economía a una ciencia natural, junto al uso/ abuso de leyes naturales a la hora de describir el funcionamiento de las economías capitalistas, considerándolo típico de los economistas vulgares y de los trabajos a más alto contenido ideológico y apologético.

En segundo lugar, reconstruimos el análisis de Antonio Gramsci del problema de la ideología en la ciencia en general y en la economía en particular, presentándolo como caso particular de la teoría marxiana, dado que profundiza y radicaliza muchas de las conclusiones de Marx. En particular, mostramos como el traspasamiento de un método de las ciencias naturales a las ciencias sociales es un error para Gramsci, debido a que la utilización la previsibilidad como criterio de verificabilidad, pero aplicado a cuestiones históricas, implica desconocer la naturaleza de que el devenir social es el resultado, en gran parte, del accionar presente. En este sentido, para Gramsci tampoco es posible hablar de objetividad de la ciencia económica porque lo objetivo o externo que se propone estudiar es el resultado de las determinaciones sociales endógenas a ese mismo fenómeno.

Finalmente, relacionamos la teoría marxiana y gramsciana al estado presente de la teoría económica. Desde esta perspectiva, destacamos que cualquier representación abstracta o matematización implica aceptar la existencia de una relación directa entre la proposición lógica y lo que esta intenta describir, y que esto no es posible en la economía ya que desconoce la naturaleza convencional e histórica de esas relaciones que se intentan analizar. Como ejemplo, consideramos la formalización en economía no fue una consecuencia natural sino que con ello voluntariamente se propuso hacer abstracción del conflicto social y otros aspectos que pudieran entrar en contradicción con la organización material de la producción. A su vez, cuestionamos la idea de progreso del conocimiento en la teoría económica ya que esta, como ámbito del "conocimiento científico", forma parte de la ideología y la superestructura y se modifica cuando cambia la base material.

En este sentido, para decirlo con Gramsci, a partir de la segunda posguerra la ciencia económica - concebida como herramienta de construcción de conocimiento - ha tenido como finalidad el establecimiento de un sentido común determinado favorable a las clases dominantes en la legitimación de la dirección del bloque histórico.

Por ello, en términos de política económica, el debate epistemológico o sobre los mecanismos de construcción de conocimiento es central. Si bien las ciertas medidas económicas son el resultado de las necesidades del sistema de producción estas, en definitiva, terminan legitimándose en relación a lo que la afirma la teoría como "correcto".

Consideramos que la actual teoría dominante por construcción metodológica de sus postulados no tiene como principal finalidad la búsqueda de un correlato empírico estrecho para describir el verdadero funcionamiento del sistema económico, sino que el eje central del análisis está puesto en el desarrollo de la formalización de sus proposiciones básicas. En este sentido, cualquier desajuste de sus predicciones con la realidad siempre puede ser atribuible a un error en las condiciones de verificabilidad explicables ad hoc manteniendo intacta los fundamentos de la teoría, produciendo un ámbito de desenvolvimiento metodológico que hace imposible cualquier cuestionamiento de la teoría dominante.

Desde este particular ángulo, es difícil no leer la economía presente como un proceso en el cual tiene cada vez más relevancia el carácter ideológico del conocimiento científico $y$, por ende, el rol que en ello juegan los economistas, modernos intelectuales encargados de construir legitimación para justificar la organización social de la producción vigente, como lo definirían Marx y Gramsci. 


\section{Referencias}

Balibar, E. (1988), The Notion of Class Politics in Marx, Rethinking Marxism, 26(1), 18-51

Cremaschi, Sergio. Ricardo and the Utilitarians. The European Journal of the History of Economic Thought 11.3 (2004): 377-403.

Davis, John B. (2002), Gramsci, Sraffa, Wittgenstein: philosophical linkages, European Journal for the History of Economic Thought, 9(3), 384-401

De Vivo, G. (2017), Nella bufera del Novecento. Antonio Gramsci e Piero Sraffa tra lotta politica e teoria critica. Roma: Castelvecchi ed.

Drucker, H.M. (1972). Marx's Concept of Ideology, Philosophy, 41(180), 152-161

Faucci, R. (2010), Croce and Gramsci as "Economists", Studi Economici, 100, 91-115

Friedman, Milton. (1953). Essays in positive economics, Chicago: Univesity of Chicago Press.

Gramsci, Antonio (1978). Americanismo e fordismo: Quaderno 22, Torino: Einaudi.

Gramsci, Antonio. (1929-1930), Punti di meditazione per lo studio dell'economia. Intorno ai Principi di Economia Pura del Pantaleoni., 10(32) http://www.gramsciproject.org/

Gramsci, Antonio. (1960). Los intelectuales y la organización de la cultura. Lautaro, 1960. Disponible en internet: http://www.ddooss.org/articulos/textos/Gramsci_Antonio.htm

Gramsci, Antonio. (1971). El materialismo histórico y la filosofía de Benedetto Croce. Buenos Aires: Nueva Visión.

Grossman, Henryk. (1977) [1941]. Marx, Classical Political Economy and the Problem of Dynamics Part I, Capital \& Class, 2, 32-55

Hempel, Carl G. and Paul Oppenheim (1948). Studies in the Logic of Explanation. Philosophy of science, $15(2), 135-175$.

Labica, Georges. (1987), Karl Marx. Les Thèses sur Feuerbach, Paris: Presses Universitaires de France.

Lampa, Roberto. (2010), Different Destinies in Assonant Circumstances: Marx's reception in Italy and Spain. 1870-1923, Pensiero Economico Italiano, 2, 11-36

Larrain, Jorge (1982), On the Character of Ideology Marx and the Present Debate in Britain, Theory, Culture \& Society, 1 (1), 5-22.

Leijonhufvud, Axel (1973). Life Among the Econ., Western Economic Journal, 11(3), 327-337.

Leopold, David (2013), Marxism and Ideology: From Marx to Althusser, in Freeden M. and Stears M. (eds.) The Oxford Handbook of Political Ideologies, Oxford University Press

Lunghini, Giorgio (1994), Scritti di economia politica di Antonio Gramsci, Roma: Bollati Boringhieri

Machlup, Fritz. (1978), Methodology of economics and other social sciences, New York: Academic Press Maguire, John (1976). Marx on ideology, power and force. Theory and Decision, 7(4), 315-329.

Marx, K and Federico Engels (1959) [1846]. La ideología alemana. Montevideo: Pueblos Unidos

Marx, Karl (1980) [1863]. Teorías sobre la Plusvalia, tomo III, Mexico DF: Fondo de Cultura Económica

Marx, Karl (1989) [1857]. Contribución a la crítica de la economía política. Buenos Aires: Siglo XXI.

Marx, Karl (2015) [1845], Tesis sobre Feuerbach, en Antología Karl Marx, Buenos Aires: Siglo XXI

Marx, Karl and Federico Engels (2009) [1894], El Capital, vol. III, Buenos Aires: Siglo XXI

Mirowski, Philip. (1989) More Heat than Light: Economics as Social Physics, Physics as Nature's Economics. Cambridge UK: Cambridge University Press.

Nielsen, Kai (1989). The Concept of Ideology: Some Marxist and Non-Marxist Conceptualizations, Rethinking Marxism, 2(4), 146-173

Parekh, Bhikhu (1982). Marx's Theory of Ideology, Texas: The John Hopkins University Press

Sen, Amartya. (2003) Sraffa, Wittgenstein, and Gramsci. Journal of Economic Literature, 41(4) 1240-1255.

Sprinzak, Ehud. (1975). Marx's Historical Conception of Ideology and Science, Politics \& Society, 5(4), $395-416$

Thwaites Rey, Mabel (2007). El Estado ampliado en el pensamiento de Gramsci. Disponible en internet: http://www.mabelthwaitesrey.com.ar/wp-content/uploads/I-Cap-4-Thwaites-Rey-Gramsci.pdf

Veblen, Thorstein (1898). Why is economics not an evolutionary science?. The Quarterly Journal of Economics, 12 (4), 373-397.

Weber, Max. (1949). The Methodology of the Social Sciences. New York: Free Press. 